Acta Crystallographica Section E

Structure Reports

Online

ISSN 1600-5368

\section{Dichlorobis[1-methylimidazoline-2(3H)-thione]- cadmium(II)}

In the title complex, $\left[\mathrm{CdCl}_{2}\left(\mathrm{C}_{4} \mathrm{H}_{6} \mathrm{~N}_{2} \mathrm{~S}\right)_{2}\right]$, the $\mathrm{Cd}$ atom has a distorted tetrahedral coordination geometry, with two $\mathrm{Cl}^{-}$and two monodentate neutral thione ligands bonded through $\mathrm{S}$. There are intramolecular $\mathrm{N}-\mathrm{H} \cdots \mathrm{Cl}$ and intermolecular $\mathrm{N}-$ $\mathrm{H}$...S hydrogen bonds, generating centrosymmetric dimers.

Department of Chemistry, Faculty of Sciences, Shahid Chamran University, Ahvaz, Iran, and

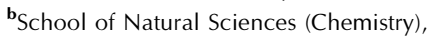

University of Newcastle upon Tyne, Newcastle upon Tyne NE1 7RU, England

Correspondence e-mail:

beheshti_a@hotmail.com,w.clegg@ncl.ac.uk

Key indicators

Single-crystal X-ray study

$T=150 \mathrm{~K}$

Mean $\sigma(\mathrm{C}-\mathrm{C})=0.004 \AA$

$R$ factor $=0.023$

$w R$ factor $=0.046$

Data-to-parameter ratio $=15.4$

For details of how these key indicators were automatically derived from the article, see http://journals.iucr.org/e.
(C) 2005 International Union of Crystallography Printed in Great Britain - all rights reserved

\section{Comment}

Due to their relevance in biological systems, the use of heterocyclic thiones as ligands in transition metal complexes has attracted much attention in the recent past (Raper, 1994, 1997), because of the search for simple model compounds for metalloproteins. In view of this, $\mathrm{Cu}^{\mathrm{I}}, \mathrm{Ag}^{\mathrm{I}}, \mathrm{Au}^{\mathrm{I}}, \mathrm{Hg}^{\mathrm{II}}$ and $\mathrm{Cd}^{\mathrm{II}}$ complexes with thiones have been widely studied (Isab et al., 2002, and references therein; Beheshti et al., 2005).<smiles>Cn1cc[nH]c1=[SH][Ge](Cl)(Cl)[SH]=c1[nH]ccn1C</smiles>

(I)

The title compound, (I), is an unexpected product, obtained in an attempt to prepare a $\mathrm{WS}_{4}(\mathrm{CdCl})_{2}(\mathrm{Hmimt})_{n}$ complex [Hmimt $=1$-methylimidazoline-2(3H)-thione $]$. The molecular structure of (I) is shown in Fig. 1, and selected bond lengths and angles are listed in Table 1 . The $\mathrm{Cd}^{\mathrm{II}}$ ion is coordinated by two $\mathrm{Cl}^{-}$and two S-bonded monodentate Hmimt ligands, to give a distorted tetrahedral $\mathrm{S}_{2} \mathrm{Cl}_{2}$ donor set. The major distortions from regular tetrahedral geometry are an enlarged

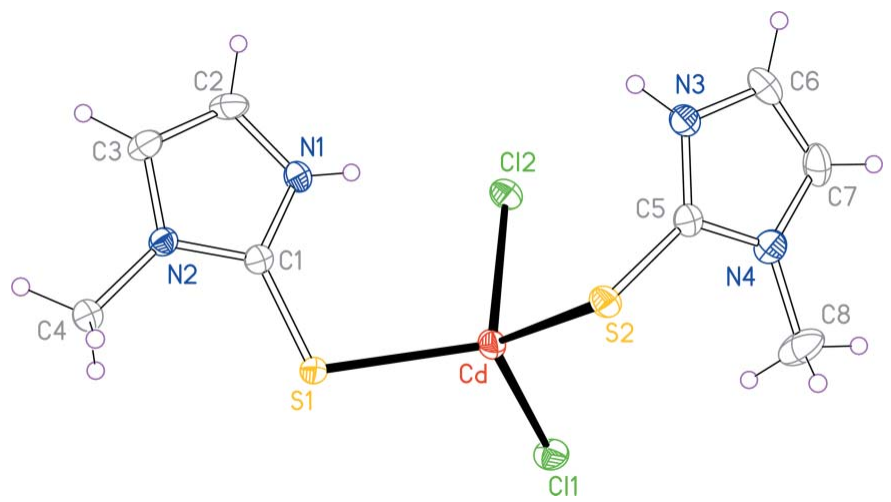

Figure 1

The molecular structure of (I), with $50 \%$ probability displacement ellipsoids.
Received 15 June 2005 Accepted 16 June 2005 Online 24 June 2005 


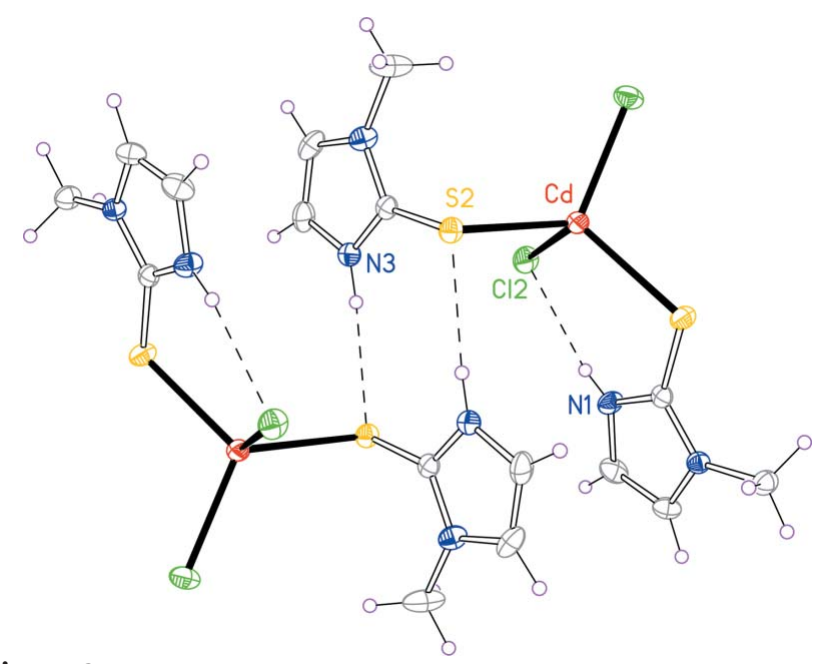

Figure 2

A centrosymmetric dimer in (I), formed by a pair of intermolecular $\mathrm{N}-$ $\mathrm{H}$...S hydrogen bonds. All hydrogen bonds are shown as dashed lines. Unlabelled atoms are generated by the symmetry operation $(-x, 1-y$, $1-z)$.

$\mathrm{S}-\mathrm{Cd}-\mathrm{S}$ angle and considerable variation in the four $\mathrm{S}-$ $\mathrm{Cd}-\mathrm{Cl}$ angles. These are probably a result mainly of steric interactions.

The essentially planar Hmimt ligands are in their neutral thione form. Their geometry is typical of this ligand attached in a monodentate fashion through $\mathrm{S}$ to metal ions; the mean $\mathrm{C}=\mathrm{S}$ bond length for almost 100 occurrences of this ligand in 39 crystal structures in the Cambridge Structural Database (version 5.26 with two updates, May 2005; Allen, 2002) is $1.718 \AA$, over a range of $1.684-1.750 \AA$ with the omission of a few outliers, and compares with $\mathrm{C}=\mathrm{S}$ bond lengths of 1.729 (3) and 1.733 (3) $\AA$ in (I). These bonds are thus lengthened and weakened on coordination, as expected, compared with their greater double-bond character in the uncomplexed ligand, which has a $\mathrm{C}=\mathrm{S}$ bond length of 1.685 (2) А (Raper et al., 1983; Vampa et al., 1995). The geometric results are in agreement with spectroscopic observations (see Experimental section).

The structure of (I) may be compared with those of other $\left[M X_{2}(\mathrm{Hmimt})_{2}\right]$ complexes $(M=\mathrm{Cd}$ or $\mathrm{Hg}$, and $X=\mathrm{Cl}, \mathrm{Br}$ or I). All of these have monomeric molecules with a distorted tetrahedral coordination geometry about the metal atom, and the variations in bond lengths and angles can be readily understood in terms of the sizes of the metal and halogen atoms. None of the other complexes is isomorphous with (I). Indeed, all five known structures have different space groups and packing arrangements (Bell et al., 2000, 2004; Pavlović et al., 2000). The bromo analogue of (I) has an unusual structure, with six molecules in the asymmetric unit and a high degree of pseudo-symmetry (Bell et al., 2004).

The $\mathrm{N}-\mathrm{H}$ groups of the two Hmimt ligands in (I) engage in hydrogen bonds. One of these is intramolecular, to atom $\mathrm{Cl} 2$, and presumably contributes to the lengthening of this $\mathrm{Cd}-\mathrm{Cl}$ bond relative to the other. The other hydrogen bond is intermolecular, to the $\mathrm{S}$ atom of an adjacent Hmimt ligand, and generates centrosymmetric dimers (Fig. 2 and Table 2).

\section{Experimental}

$\mathrm{CdCl}_{2} \cdot \mathrm{H}_{2} \mathrm{O}(1.12 \mathrm{~g}, 5.56 \mathrm{mmol})$ was added to a suspension of $\left(\mathrm{NH}_{4}\right)_{2}\left[\mathrm{WS}_{4}\right](0.967 \mathrm{~g}, 2.78 \mathrm{mmol})$ in acetone $(70 \mathrm{ml})$ and the mixture was stirred for $1 \mathrm{~h}$. Hmimt $(0.71 \mathrm{~g}, 6.22 \mathrm{mmol})$ was added to this solution and the mixture was stirred for another $4 \mathrm{~h}$ at room temperature. The mixture was centrifuged and the yellow supernatant liquid was decanted and evaporated to dryness in a vacuum. The residue was washed with diethyl ether $(2 \times 5 \mathrm{ml})$ and $n$-pentane $(2 \times 5 \mathrm{ml})$ to remove any unreacted Hmimt, and dried in a vacuum to give an orange-yellow powder. Both IR $\left[v(\mathrm{~W}-\mathrm{S})=441 \mathrm{~cm}^{-1}\right.$, $v(\mathrm{C}=\mathrm{S})=506 \mathrm{~cm}^{-1}$ and $\nu(\mathrm{N}-\mathrm{H})=3133 \mathrm{~cm}^{-1}$ ] and UV-vis $\lambda_{\max }=$ $430,374,315$ and $273 \mathrm{~nm}$ ) spectra of the product confirmed the presence of $\mathrm{WS}_{4}$ and S-bonded Hmimt ligands. In the solid state, the complex is air-stable and can be stored for months in a desiccator, but it decomposed slowly when diethyl ether was diffused slowly into an acetone solution of the product over $3 \mathrm{~d}$ at room temperature, resulting in the formation of pale-yellow crystals with an empirical formula $\mathrm{C}_{8} \mathrm{H}_{12} \mathrm{CdCl}_{2} \mathrm{~N}_{4} \mathrm{~S}_{2}$, as confirmed by X-ray crystallography. The air-stable crystals of this compound are insoluble in common organic solvents, but soluble in solvents with pronounced donor properties, such as dimethyl sulfoxide and dimethylformamide. ${ }^{1} \mathrm{H}$ NMR (DMSO- $d_{6}, 298 \mathrm{~K}, \delta$, p.p.m.): $12.01(s, \mathrm{NH}), 6.98(s, \mathrm{CH}), 6.80(s, \mathrm{CH})$, $3.32\left(s, \mathrm{NCH}_{3}\right) ;{ }^{13} \mathrm{C} \mathrm{NMR}$ (DMSO- $d_{6}, 298 \mathrm{~K}, \delta$, p.p.m.): $160.52(\mathrm{C} 1 / 5)$, 120.05 and 114.62 (C2/6 and C3/7), 33.99 (C4/8) (see Fig. 1 for atom numbering). In the NMR spectra of the complex, the ligand signals are shifted down-field from their positions in the spectra of the free ligand (Casa et al., 1996), suggesting that, in DMSO- $d_{6}$, the ligand remains coordinated to the metal. The absence of a weak $\mathrm{S}-\mathrm{H}$ signal of the thiol form of the ligand in the ${ }^{1} \mathrm{H}$ NMR spectrum of the complex confirms that coordination of Hmimt in DMSO- $d_{6}$ solution, as in the solid state, takes place only through the S atom, the Hmimt ligands being in the neutral thione form.

\section{Crystal data}

$\left[\mathrm{CdCl}_{2}\left(\mathrm{C}_{4} \mathrm{H}_{6} \mathrm{~N}_{2} \mathrm{~S}\right)_{2}\right]$

$M_{r}=411.64$

Monoclinic, $P 2_{1} / c$

$a=9.6464(8) \AA$

$b=7.6262(8) \AA$

$c=19.7151(8) \AA$

$\beta=96.485(6)^{\circ}$

$V=1441.1(2) \AA^{3}$

$Z=4$

\section{Data collection}

Nonius KappaCCD area-detector diffractometer

$\varphi$ and $\omega$ scans

Absorption correction: multi-scan (SADABS; Sheldrick, 2002)

$T_{\min }=0.481, T_{\max }=0.549$

22587 measured reflections

\section{Refinement}

Refinement on $F^{2}$

$R\left[F^{2}>2 \sigma\left(F^{2}\right)\right]=0.023$

$w R\left(F^{2}\right)=0.046$

$S=1.05$

2518 reflections

163 parameters

$\mathrm{H}$ atoms treated by a mixture of independent and constrained refinement

\author{
$D_{x}=1.897 \mathrm{Mg} \mathrm{m}^{-3}$ \\ Mo $K \alpha$ radiation \\ Cell parameters from 22587 \\ reflections \\ $\theta=2.5-25.0^{\circ}$ \\ $\mu=2.16 \mathrm{~mm}^{-1}$ \\ $T=150$ (2) K \\ Block, pale yellow \\ $0.36 \times 0.32 \times 0.28 \mathrm{~mm}$
}

2518 independent reflections 2115 reflections with $I>2 \sigma(I)$

$R_{\text {int }}=0.058$

$\theta_{\text {max }}=25.0^{\circ}$

$h=-11 \rightarrow 11$

$k=-9 \rightarrow 9$

$l=-23 \rightarrow 23$

$w=1 /\left[\sigma^{2}\left(F_{\mathrm{o}}{ }^{2}\right)+(0.016 P)^{2}\right.$ $+0.9012 P]$

where $P=\left(F_{\mathrm{o}}{ }^{2}+2 F_{\mathrm{c}}{ }^{2}\right) / 3$

$(\Delta / \sigma)_{\max }=0.001$

$\Delta \rho_{\max }=0.36{\mathrm{e} \AA^{-3}}^{-3}$

$\Delta \rho_{\min }=-0.32 \mathrm{e}^{-3}$

Extinction correction: SHELXTL (Sheldrick, 2001)

Extinction coefficient: 0.0027 (3) 
Table 1

Selected geometric parameters $\left(\AA{ }^{\circ}\right)$.

\begin{tabular}{lllr}
\hline $\mathrm{Cd}-\mathrm{Cl} 1$ & $2.4410(7)$ & $\mathrm{Cd}-\mathrm{S} 2$ & $2.5663(7)$ \\
$\mathrm{Cd}-\mathrm{Cl} 2$ & $2.5175(7)$ & $\mathrm{S} 1-\mathrm{C} 1$ & $1.729(3)$ \\
$\mathrm{Cd}-\mathrm{S} 1$ & $2.5392(7)$ & $\mathrm{S} 2-\mathrm{C} 5$ & $1.733(3)$ \\
& & & \\
$\mathrm{Cl} 1-\mathrm{Cd}-\mathrm{Cl} 2$ & $104.43(2)$ & $\mathrm{Cl} 2-\mathrm{Cd}-\mathrm{S} 2$ & $97.66(2)$ \\
$\mathrm{Cl} 1-\mathrm{Cd}-\mathrm{S} 1$ & $111.60(2)$ & $\mathrm{S} 1-\mathrm{Cd}-\mathrm{S} 2$ & $117.32(2)$ \\
$\mathrm{Cl} 1-\mathrm{Cd}-\mathrm{S} 2$ & $116.52(2)$ & $\mathrm{Cd}-\mathrm{S} 1-\mathrm{C} 1$ & $107.78(9)$ \\
$\mathrm{Cl} 2-\mathrm{Cd}-\mathrm{S} 1$ & $106.84(2)$ & $\mathrm{Cd}-\mathrm{S} 2-\mathrm{C} 5$ & $102.85(9)$ \\
\hline
\end{tabular}

Table 2

Hydrogen-bond geometry $\left(\AA{ }^{\circ}\right)$.

\begin{tabular}{lllll}
\hline$D-\mathrm{H} \cdots A$ & $D-\mathrm{H}$ & $\mathrm{H} \cdots A$ & $D \cdots A$ & $D-\mathrm{H} \cdots A$ \\
\hline $\mathrm{N} 1-\mathrm{H} 1 \cdots \mathrm{Cl} 2$ & $0.80(3)$ & $2.41(3)$ & $3.204(3)$ & $168(3)$ \\
$\mathrm{N} 3-\mathrm{H} 3 \cdots \mathrm{S} 2{ }^{\mathrm{i}}$ & $0.88(3)$ & $2.49(3)$ & $3.358(3)$ & $173(3)$
\end{tabular}

Symmetry codes: (i) $-x,-y+1,-z+1$.

All $\mathrm{H}$ atoms were located in a difference map. Those bonded to $\mathrm{N}$ were refined with unconstrained coordinates and $U_{\text {iso }}(\mathrm{H})=$ $1.2 U_{\text {eq }}(\mathrm{N})$. Other $\mathrm{H}$ atoms were refined as riding with idealized geometries, including free rotation of methyl groups about the $\mathrm{C}-\mathrm{C}$ bonds $(\mathrm{C}-\mathrm{H}=0.95-0.98 \AA)$, with the constraint $U_{\text {iso }}(\mathrm{H})=1.2 U_{\text {eq }}(\mathrm{C})$ or $1.5 U_{\text {eq }}$ (methyl C) applied.

Data collection: COLLECT (Nonius, 1998); cell refinement: EVALCCD (Duisenberg et al., 2003); data reduction: EVALCCD; program(s) used to solve structure: SHELXTL (Sheldrick, 2001); program(s) used to refine structure: SHELXTL; molecular graphics: SHELXTL; software used to prepare material for publication: SHELXTL and local programs.

The authors thank the EPSRC (UK) and Shahid Chamran University for financial support.

\section{References}

Allen, F. H. (2002). Acta Cryst. B58, 380-388.

Beheshti, A., Clegg, W., Brooks, N. R. \& Sharafi, F. (2005). Polyhedron, 24 435-441.

Bell, N. A., Branston, T. N., Clegg, W., Creighton, J. R., Cucurull-Sanchez, L., Elsegood, M. R. J. \& Raper, E. S. (2000). Inorg. Chim. Acta, 303, 220-227.

Bell, N. A., Clegg, W., Coles, S. J., Constable, C. P., Harrington, R. W., Hursthouse, M. B., Light, M. E., Raper, E. S., Sammon, C. \& Walker, M. R. (2004). Inorg. Chim. Acta, 357, 2091-2099.

Casa, J. S., Martinez, E. G., Sánchez, A., González, A. S., Sordo, J., Casellato, U. \& Graziani, R. (1996). Inorg. Chim. Acta, 241, 117-123.

Duisenberg, A. J. M., Kroon-Batenburg, L. M. J. \& Schreurs, A. M. M. (2003). J. Appl. Cryst. 36, 220-229.

Isab, A. A., Ahmad, S. \& Arab, M. (2002). Polyhedron, 21, 1267-1271.

Nonius (1998). COLLECT. Nonius BV, Delft, The Netherlands.

Pavlović, G., Popović, Z., Soldin, Ž. \& Matković-Čalogović, D. (2000). Acta Cryst. C56, 801-803.

Raper, E. S. (1994). Coord. Chem. Rev. 129, 91-156.

Raper, E. S. (1997). Coord. Chem. Rev. 165, 475-567.

Raper, E. S., Creighton, J. R., Oughtred, R. E. \& Nowell, I. W. (1983). Acta Cryst. B39, 355-360.

Sheldrick, G. M. (2001). SHELXTL. Version 6.10. Bruker AXS Inc., Madison, Wisconsin, USA.

Sheldrick, G. M. (2002). SADABS. Version 2.06. University of Göttingen, Germany.

Vampa, G., Benvenuti, S., Severi, F., Malmusi, L. \& Antolini, L. (1995). J. Heterocycl. Chem. 32, 227-234. 


\section{supporting information}

Acta Cryst. (2005). E61, m1383-m1385 [https://doi.org/10.1107/S1600536805019203]

\section{Dichlorobis[1-methylimidazoline-2(3H)-thione] cadmium(II)}

\section{Azizolla Beheshti, Neil R. Brooks, William Clegg and Reza Hyvadi}

Dichlorobis[1-methylimidazoline-2(3H)-thione]cadmium(II)

Crystal data

$\left[\mathrm{CdCl}_{2}\left(\mathrm{C}_{4} \mathrm{H}_{6} \mathrm{~N}_{2} \mathrm{~S}\right)_{2}\right]$

$M_{r}=411.64$

Monoclinic, $P 2_{1} / c$

Hall symbol: -P 2ybc

$a=9.6464(8) \AA$

$b=7.6262(8) \AA$

$c=19.7151(8) \AA$

$\beta=96.485(6)^{\circ}$

$V=1441.1(2) \AA^{3}$

$Z=4$

\section{Data collection}

Nonius KappaCCD area-detector diffractometer

Radiation source: sealed tube

Graphite monochromator

$\varphi$ and $\omega$ scans

Absorption correction: multi-scan

(SADABS; Sheldrick, 2002)

$T_{\min }=0.481, T_{\max }=0.549$

\section{Refinement}

Refinement on $F^{2}$

Least-squares matrix: full

$R\left[F^{2}>2 \sigma\left(F^{2}\right)\right]=0.023$

$w R\left(F^{2}\right)=0.046$

$S=1.05$

2518 reflections

163 parameters

0 restraints

Primary atom site location: structure-invariant direct methods

Secondary atom site location: difference Fourier map
$F(000)=808$

$D_{\mathrm{x}}=1.897 \mathrm{Mg} \mathrm{m}^{-3}$

Mo $K \alpha$ radiation, $\lambda=0.71073 \AA$

Cell parameters from 23643 reflections

$\theta=2.5-25.0^{\circ}$

$\mu=2.16 \mathrm{~mm}^{-1}$

$T=150 \mathrm{~K}$

Block, pale yellow

$0.36 \times 0.32 \times 0.28 \mathrm{~mm}$

22587 measured reflections

2518 independent reflections

2115 reflections with $I>2 \sigma(I)$

$R_{\text {int }}=0.058$

$\theta_{\max }=25.0^{\circ}, \theta_{\min }=4.9^{\circ}$

$h=-11 \rightarrow 11$

$k=-9 \rightarrow 9$

$l=-23 \rightarrow 23$

Hydrogen site location: inferred from neighbouring sites

$\mathrm{H}$ atoms treated by a mixture of independent and constrained refinement

$w=1 /\left[\sigma^{2}\left(F_{\mathrm{o}}{ }^{2}\right)+(0.016 P)^{2}+0.9012 P\right]$ where $P=\left(F_{\mathrm{o}}{ }^{2}+2 F_{\mathrm{c}}{ }^{2}\right) / 3$

$(\Delta / \sigma)_{\max }=0.001$

$\Delta \rho_{\max }=0.36 \mathrm{e} \AA^{-3}$

$\Delta \rho_{\min }=-0.32$ e $\AA^{-3}$

Extinction correction: SHELXTL (Sheldrick, 2001), $\mathrm{Fc}^{*}=\mathrm{kFc}\left[1+0.001 \mathrm{xFc}^{2} \lambda^{3} / \sin (2 \theta)\right]^{-1 / 4}$

Extinction coefficient: 0.0027 (3)

Fractional atomic coordinates and isotropic or equivalent isotropic displacement parameters $\left(\AA^{2}\right)$

\begin{tabular}{lllll}
\hline & $x$ & $y$ & $z$ & $U_{\text {iso }} * U_{\text {eq }}$ \\
\hline $\mathrm{Cd}$ & $0.305327(19)$ & $0.40552(3)$ & $0.413963(10)$ & $0.01882(9)$
\end{tabular}




$\begin{array}{lllll}\text { C11 } & 0.41103(7) & 0.42594(9) & 0.30744(3) & 0.02480(17) \\ \text { C12 } & 0.11347(7) & 0.18284(9) & 0.38971(3) & 0.02436(17) \\ \text { S1 } & 0.47501(7) & 0.28210(9) & 0.50996(3) & 0.02039(17) \\ \text { C1 } & 0.3861(3) & 0.1299(3) & 0.55362(13) & 0.0159(6) \\ \text { N1 } & 0.2581(2) & 0.0623(3) & 0.53657(12) & 0.0206(6) \\ \text { H1 } & 0.211(3) & 0.084(4) & 0.5012(16) & 0.025^{*} \\ \text { N2 } & 0.4360(2) & 0.0558(3) & 0.61350(11) & 0.0165(5) \\ \text { C2 } & 0.2271(3) & -0.0553(4) & 0.58620(14) & 0.0246(7) \\ \text { H2 } & 0.1434 & -0.1210 & 0.5863 & 0.030^{*} \\ \text { C3 } & 0.3374(3) & -0.0588(3) & 0.63401(14) & 0.0216(6) \\ \text { H3A } & 0.3463 & -0.1272 & 0.6745 & 0.026^{*} \\ \text { C4 } & 0.5738(3) & 0.0896(4) & 0.65069(14) & 0.0216(6) \\ \text { H4A } & 0.6455 & 0.0766 & 0.6196 & 0.032^{*} \\ \text { H4B } & 0.5914 & 0.0056 & 0.6883 & 0.032^{*} \\ \text { H4C } & 0.5766 & 0.2091 & 0.6690 & 0.032^{*} \\ \text { S2 } & 0.15259(7) & 0.66470(9) & 0.44266(3) & 0.01838(17) \\ \text { C5 } & -0.0033(3) & 0.6253(3) & 0.39218(13) & 0.0165(6) \\ \text { N3 } & -0.1171(2) & 0.5455(3) & 0.41061(12) & 0.0205(5) \\ \text { H3 } & -0.119(3) & 0.495(4) & 0.4504(16) & 0.025^{*} \\ \text { N4 } & -0.0372(2) & 0.6812(3) & 0.32768(11) & 0.0202(5) \\ \text { C6 } & -0.2242(3) & 0.5515(4) & 0.35804(15) & 0.0255(7) \\ \text { H6 } & -0.3154 & 0.5045 & 0.3583 & 0.031^{*} \\ \text { C7 } & -0.1741(3) & 0.6372(4) & 0.30602(15) & 0.0257(7) \\ \text { H7 } & -0.2236 & 0.6624 & 0.2627 & 0.031^{*} \\ \text { C8 } & 0.0581(3) & 0.7743(4) & 0.28699(15) & 0.0351(8) \\ \text { H8A } & 0.0866 & 0.8854 & 0.3093 & 0.053^{*} \\ \text { H8B } & 0.0105 & 0.7978 & 0.2413 & 0.053^{*} \\ \text { H8C } & 0.1407 & 0.7017 & 0.2832 & 0.053^{*} \\ & & & & \end{array}$

Atomic displacement parameters $\left(\AA^{2}\right)$

\begin{tabular}{lllllll}
\hline & $U^{11}$ & $U^{22}$ & $U^{33}$ & $U^{12}$ & $U^{13}$ & $U^{23}$ \\
\hline Cd & $0.01600(12)$ & $0.02447(13)$ & $0.01584(13)$ & $0.00119(9)$ & $0.00109(8)$ & $0.00265(9)$ \\
C11 & $0.0252(4)$ & $0.0334(4)$ & $0.0164(4)$ & $-0.0041(3)$ & $0.0050(3)$ & $0.0002(3)$ \\
C12 & $0.0185(4)$ & $0.0279(4)$ & $0.0256(4)$ & $-0.0043(3)$ & $-0.0022(3)$ & $0.0001(3)$ \\
S1 & $0.0164(3)$ & $0.0233(4)$ & $0.0205(4)$ & $-0.0050(3)$ & $-0.0019(3)$ & $0.0071(3)$ \\
C1 & $0.0173(14)$ & $0.0131(15)$ & $0.0177(15)$ & $0.0013(11)$ & $0.0031(11)$ & $-0.0008(11)$ \\
N1 & $0.0180(13)$ & $0.0261(15)$ & $0.0164(13)$ & $-0.0042(10)$ & $-0.0041(10)$ & $0.0018(10)$ \\
N2 & $0.0173(12)$ & $0.0156(12)$ & $0.0166(13)$ & $-0.0011(9)$ & $0.0021(10)$ & $0.0010(9)$ \\
C2 & $0.0217(16)$ & $0.0268(17)$ & $0.0263(17)$ & $-0.0099(12)$ & $0.0067(13)$ & $-0.0010(13)$ \\
C3 & $0.0258(16)$ & $0.0209(16)$ & $0.0189(15)$ & $-0.0055(12)$ & $0.0056(13)$ & $0.0015(12)$ \\
C4 & $0.0195(15)$ & $0.0253(16)$ & $0.0194(15)$ & $-0.0028(13)$ & $-0.0009(12)$ & $0.0026(12)$ \\
S2 & $0.0159(3)$ & $0.0204(4)$ & $0.0182(4)$ & $-0.0020(3)$ & $-0.0012(3)$ & $-0.0011(3)$ \\
C5 & $0.0183(14)$ & $0.0138(15)$ & $0.0173(15)$ & $0.0018(11)$ & $0.0014(11)$ & $-0.0024(11)$ \\
N3 & $0.0170(13)$ & $0.0255(14)$ & $0.0192(13)$ & $0.0018(10)$ & $0.0022(11)$ & $0.0028(10)$ \\
N4 & $0.0234(13)$ & $0.0196(13)$ & $0.0165(13)$ & $-0.0046(10)$ & $-0.0026(10)$ & $-0.0011(10)$ \\
C6 & $0.0154(15)$ & $0.0305(18)$ & $0.0291(17)$ & $0.0025(12)$ & $-0.0037(13)$ & $-0.0036(13)$ \\
C7 & $0.0272(16)$ & $0.0229(17)$ & $0.0241(16)$ & $0.0031(13)$ & $-0.0098(13)$ & $0.0002(13)$
\end{tabular}




$\begin{array}{lllllll}\mathrm{C} 8 & 0.0445(19) & 0.041(2) & 0.0185(17) & -0.0199(16) & -0.0006(14) & 0.0072(14)\end{array}$

Geometric parameters $(\AA, \stackrel{o}{)}$

\begin{tabular}{|c|c|c|c|}
\hline $\mathrm{Cd}-\mathrm{Cl1}$ & $2.4410(7)$ & $\mathrm{C} 4-\mathrm{H} 4 \mathrm{~B}$ & 0.980 \\
\hline $\mathrm{Cd}-\mathrm{Cl} 2$ & $2.5175(7)$ & $\mathrm{C} 4-\mathrm{H} 4 \mathrm{C}$ & 0.980 \\
\hline $\mathrm{Cd}-\mathrm{S} 1$ & $2.5392(7)$ & $\mathrm{S} 2-\mathrm{C} 5$ & $1.733(3)$ \\
\hline $\mathrm{Cd}-\mathrm{S} 2$ & $2.5663(7)$ & $\mathrm{C} 5-\mathrm{N} 3$ & $1.341(3)$ \\
\hline $\mathrm{S} 1-\mathrm{C} 1$ & $1.729(3)$ & $\mathrm{C} 5-\mathrm{N} 4$ & $1.346(3)$ \\
\hline $\mathrm{C} 1-\mathrm{N} 1$ & $1.345(3)$ & N3-H3 & $0.88(3)$ \\
\hline $\mathrm{C} 1-\mathrm{N} 2$ & $1.348(3)$ & N3-C6 & $1.379(4)$ \\
\hline $\mathrm{N} 1-\mathrm{H} 1$ & $0.80(3)$ & $\mathrm{N} 4-\mathrm{C} 7$ & $1.383(4)$ \\
\hline $\mathrm{N} 1-\mathrm{C} 2$ & $1.385(4)$ & $\mathrm{N} 4-\mathrm{C} 8$ & $1.469(4)$ \\
\hline $\mathrm{N} 2-\mathrm{C} 3$ & $1.386(3)$ & C6- 6 6 & 0.950 \\
\hline $\mathrm{N} 2-\mathrm{C} 4$ & $1.466(3)$ & $\mathrm{C} 6-\mathrm{C} 7$ & $1.351(4)$ \\
\hline $\mathrm{C} 2-\mathrm{H} 2$ & 0.950 & $\mathrm{C} 7-\mathrm{H} 7$ & 0.950 \\
\hline $\mathrm{C} 2-\mathrm{C} 3$ & $1.340(4)$ & $\mathrm{C} 8-\mathrm{H} 8 \mathrm{~A}$ & 0.980 \\
\hline $\mathrm{C} 3-\mathrm{H} 3 \mathrm{~A}$ & 0.950 & $\mathrm{C} 8-\mathrm{H} 8 \mathrm{~B}$ & 0.980 \\
\hline $\mathrm{C} 4-\mathrm{H} 4 \mathrm{~A}$ & 0.980 & $\mathrm{C} 8-\mathrm{H} 8 \mathrm{C}$ & 0.980 \\
\hline $\mathrm{Cl} 1-\mathrm{Cd}-\mathrm{Cl} 2$ & $104.43(2)$ & $\mathrm{H} 4 \mathrm{~A}-\mathrm{C} 4-\mathrm{H} 4 \mathrm{~B}$ & 109.5 \\
\hline $\mathrm{C} 11-\mathrm{Cd}-\mathrm{S} 1$ & $111.60(2)$ & $\mathrm{H} 4 \mathrm{~A}-\mathrm{C} 4-\mathrm{H} 4 \mathrm{C}$ & 109.5 \\
\hline $\mathrm{C} 11-\mathrm{Cd}-\mathrm{S} 2$ & $116.52(2)$ & $\mathrm{H} 4 \mathrm{~B}-\mathrm{C} 4-\mathrm{H} 4 \mathrm{C}$ & 109.5 \\
\hline $\mathrm{Cl} 2-\mathrm{Cd}-\mathrm{S} 1$ & $106.84(2)$ & $\mathrm{Cd}-\mathrm{S} 2-\mathrm{C} 5$ & $102.85(9)$ \\
\hline $\mathrm{Cl} 2-\mathrm{Cd}-\mathrm{S} 2$ & $97.66(2)$ & $\mathrm{S} 2-\mathrm{C} 5-\mathrm{N} 3$ & $127.4(2)$ \\
\hline $\mathrm{S} 1-\mathrm{Cd}-\mathrm{S} 2$ & $117.32(2)$ & $\mathrm{S} 2-\mathrm{C} 5-\mathrm{N} 4$ & $126.1(2)$ \\
\hline $\mathrm{Cd}-\mathrm{S} 1-\mathrm{C} 1$ & $107.78(9)$ & $\mathrm{N} 3-\mathrm{C} 5-\mathrm{N} 4$ & $106.3(2)$ \\
\hline $\mathrm{S} 1-\mathrm{C} 1-\mathrm{N} 1$ & $128.8(2)$ & $\mathrm{C} 5-\mathrm{N} 3-\mathrm{H} 3$ & $123.1(19)$ \\
\hline $\mathrm{S} 1-\mathrm{C} 1-\mathrm{N} 2$ & $124.7(2)$ & $\mathrm{C} 5-\mathrm{N} 3-\mathrm{C} 6$ & $110.4(2)$ \\
\hline $\mathrm{N} 1-\mathrm{C} 1-\mathrm{N} 2$ & $106.6(2)$ & $\mathrm{H} 3-\mathrm{N} 3-\mathrm{C} 6$ & 126.5 (19) \\
\hline $\mathrm{C} 1-\mathrm{N} 1-\mathrm{H} 1$ & $123(2)$ & $\mathrm{C} 5-\mathrm{N} 4-\mathrm{C} 7$ & $109.6(2)$ \\
\hline $\mathrm{C} 1-\mathrm{N} 1-\mathrm{C} 2$ & $109.7(2)$ & $\mathrm{C} 5-\mathrm{N} 4-\mathrm{C} 8$ & $124.7(2)$ \\
\hline $\mathrm{H} 1-\mathrm{N} 1-\mathrm{C} 2$ & $127(2)$ & $\mathrm{C} 7-\mathrm{N} 4-\mathrm{C} 8$ & $125.7(2)$ \\
\hline $\mathrm{C} 1-\mathrm{N} 2-\mathrm{C} 3$ & $109.3(2)$ & $\mathrm{N} 3-\mathrm{C} 6-\mathrm{H} 6$ & 126.7 \\
\hline $\mathrm{C} 1-\mathrm{N} 2-\mathrm{C} 4$ & $125.3(2)$ & $\mathrm{N} 3-\mathrm{C} 6-\mathrm{C} 7$ & $106.5(3)$ \\
\hline $\mathrm{C} 3-\mathrm{N} 2-\mathrm{C} 4$ & $125.3(2)$ & $\mathrm{H} 6-\mathrm{C} 6-\mathrm{C} 7$ & 126.7 \\
\hline $\mathrm{N} 1-\mathrm{C} 2-\mathrm{H} 2$ & 126.5 & $\mathrm{~N} 4-\mathrm{C} 7-\mathrm{C} 6$ & $107.1(3)$ \\
\hline $\mathrm{N} 1-\mathrm{C} 2-\mathrm{C} 3$ & $107.0(2)$ & $\mathrm{N} 4-\mathrm{C} 7-\mathrm{H} 7$ & 126.4 \\
\hline $\mathrm{H} 2-\mathrm{C} 2-\mathrm{C} 3$ & 126.5 & $\mathrm{C} 6-\mathrm{C} 7-\mathrm{H} 7$ & 126.4 \\
\hline $\mathrm{N} 2-\mathrm{C} 3-\mathrm{C} 2$ & $107.4(2)$ & $\mathrm{N} 4-\mathrm{C} 8-\mathrm{H} 8 \mathrm{~A}$ & 109.5 \\
\hline $\mathrm{N} 2-\mathrm{C} 3-\mathrm{H} 3 \mathrm{~A}$ & 126.3 & $\mathrm{~N} 4-\mathrm{C} 8-\mathrm{H} 8 \mathrm{~B}$ & 109.5 \\
\hline $\mathrm{C} 2-\mathrm{C} 3-\mathrm{H} 3 \mathrm{~A}$ & 126.3 & $\mathrm{~N} 4-\mathrm{C} 8-\mathrm{H} 8 \mathrm{C}$ & 109.5 \\
\hline $\mathrm{N} 2-\mathrm{C} 4-\mathrm{H} 4 \mathrm{~A}$ & 109.5 & $\mathrm{H} 8 \mathrm{~A}-\mathrm{C} 8-\mathrm{H} 8 \mathrm{~B}$ & 109.5 \\
\hline $\mathrm{N} 2-\mathrm{C} 4-\mathrm{H} 4 \mathrm{~B}$ & 109.5 & $\mathrm{H} 8 \mathrm{~A}-\mathrm{C} 8-\mathrm{H} 8 \mathrm{C}$ & 109.5 \\
\hline $\mathrm{N} 2-\mathrm{C} 4-\mathrm{H} 4 \mathrm{C}$ & 109.5 & $\mathrm{H} 8 \mathrm{~B}-\mathrm{C} 8-\mathrm{H} 8 \mathrm{C}$ & 109.5 \\
\hline $\mathrm{C} 11-\mathrm{Cd}-\mathrm{S} 1-\mathrm{C} 1$ & $-136.06(9)$ & $\mathrm{C} 11-\mathrm{Cd}-\mathrm{S} 2-\mathrm{C} 5$ & $79.26(10)$ \\
\hline $\mathrm{Cl} 2-\mathrm{Cd}-\mathrm{S} 1-\mathrm{C} 1$ & $-22.49(10)$ & $\mathrm{Cl} 2-\mathrm{Cd}-\mathrm{S} 2-\mathrm{C} 5$ & $-31.11(9)$ \\
\hline
\end{tabular}




$\begin{array}{ll}\mathrm{S} 2-\mathrm{Cd}-\mathrm{S} 1-\mathrm{C} 1 & 85.76(10) \\ \mathrm{Cd}-\mathrm{S} 1-\mathrm{C} 1-\mathrm{N} 1 & 10.0(3) \\ \mathrm{Cd}-\mathrm{S} 1-\mathrm{C} 1-\mathrm{N} 2 & -169.9(2) \\ \mathrm{S} 1-\mathrm{C} 1-\mathrm{N} 1-\mathrm{C} 2 & -179.8(2) \\ \mathrm{N} 2-\mathrm{C} 1-\mathrm{N} 1-\mathrm{C} 2 & 0.1(3) \\ \mathrm{S} 1-\mathrm{C} 1-\mathrm{N} 2-\mathrm{C} 3 & 179.65(19) \\ \mathrm{S} 1-\mathrm{C} 1-\mathrm{N} 2-\mathrm{C} 4 & -1.0(4) \\ \mathrm{N} 1-\mathrm{C} 1-\mathrm{N} 2-\mathrm{C} 3 & -0.3(3) \\ \mathrm{N} 1-\mathrm{C} 1-\mathrm{N} 2-\mathrm{C} 4 & 179.0(2) \\ \mathrm{C} 1-\mathrm{N} 1-\mathrm{C} 2-\mathrm{C} 3 & 0.1(3) \\ \mathrm{N} 1-\mathrm{C} 2-\mathrm{C} 3-\mathrm{N} 2 & -0.3(3) \\ \mathrm{C} 1-\mathrm{N} 2-\mathrm{C} 3-\mathrm{C} 2 & 0.4(3) \\ \mathrm{C} 4-\mathrm{N} 2-\mathrm{C} 3-\mathrm{C} 2 & -179.0(2)\end{array}$

$\mathrm{S} 1-\mathrm{Cd}-\mathrm{S} 2-\mathrm{C} 5$

$\mathrm{Cd}-\mathrm{S} 2-\mathrm{C} 5-\mathrm{N} 3$

$\mathrm{Cd}-\mathrm{S} 2-\mathrm{C} 5-\mathrm{N} 4$

$\mathrm{S} 2-\mathrm{C} 5-\mathrm{N} 3-\mathrm{C} 6$

$\mathrm{N} 4-\mathrm{C} 5-\mathrm{N} 3-\mathrm{C} 6$

$\mathrm{S} 2-\mathrm{C} 5-\mathrm{N} 4-\mathrm{C} 7$

$\mathrm{S} 2-\mathrm{C} 5-\mathrm{N} 4-\mathrm{C} 8$

$\mathrm{N} 3-\mathrm{C} 5-\mathrm{N} 4-\mathrm{C} 7$

$\mathrm{N} 3-\mathrm{C} 5-\mathrm{N} 4-\mathrm{C} 8$

$\mathrm{C} 5-\mathrm{N} 3-\mathrm{C} 6-\mathrm{C} 7$

N3- $6-\mathrm{C} 7-\mathrm{N} 4$

$\mathrm{C} 5-\mathrm{N} 4-\mathrm{C} 7-\mathrm{C} 6$

$\mathrm{C} 8-\mathrm{N} 4-\mathrm{C} 7-\mathrm{C} 6$
$-144.60(9)$

$96.2(2)$

$-89.2(2)$

$175.1(2)$

-0.4 (3)

$-175.0(2)$

$5.2(4)$

$0.6(3)$

$-179.2(3)$

$0.1(3)$

$0.3(3)$

$-0.5(3)$

$179.3(3)$

Hydrogen-bond geometry $\left(\AA,{ }^{\circ}\right)$

\begin{tabular}{lllll}
\hline$D-\mathrm{H} \cdots A$ & $D-\mathrm{H}$ & $\mathrm{H} \cdots A$ & $D \cdots A$ & $D-\mathrm{H} \cdots A$ \\
\hline $\mathrm{N} 1-\mathrm{H} 1 \cdots \mathrm{Cl} 2$ & $0.80(3)$ & $2.41(3)$ & $3.204(3)$ & $168(3)$ \\
$\mathrm{N} 3-\mathrm{H} 3 \cdots \mathrm{S} 2^{\mathrm{i}}$ & $0.88(3)$ & $2.49(3)$ & $3.358(3)$ & $173(3)$ \\
\hline
\end{tabular}

Symmetry code: (i) $-x,-y+1,-z+1$. 